\title{
Active quadrupole stabilization for future linear particle colliders
}

\author{
C. Collette*, K. Artoos, A. Kuzmin, S. Janssens, M. Sylte, M. Guinchard, C. Hauviller \\ Engineering Department, European Organization for Nuclear Research, Geneva 23, 1211 Geneva, Switzerland
}

\section{A R T I C L E I N F O}

\section{Article history:}

Received 16 December 2009

Received in revised form

29 April 2010

Accepted 8 May 2010

Available online 16 May 2010

Keywords:

Quadrupole stabilization

Parallel robot

Hexapod

SVD controller

Nano-positioning

\begin{abstract}
A B S T R A C T
The future Compact LInear particle Collider (CLIC) under study at CERN will require to stabilize heavy electromagnets, and also to provide them some positioning capabilities. Firstly, this paper presents the concept adopted to address both requirements. Secondly, the control strategy adopted for the stabilization is studied numerically, showing that the quadrupole can be stabilized in both lateral and vertical direction. Finally, the strategy is validated experimentally on a single degree of freedom scaled test bench.
\end{abstract}

(c) 2010 Elsevier B.V. All rights reserved.

\section{Introduction}

In the Compact LInear Collider (CLIC) currently under study [1], electrons and positrons will be accelerated in two linear accelerators to collide at the interaction point with an energy of 0.5-3 TeV. To acquire such a high energy, the total length of the machine should to be up to $48 \mathrm{~km}$. This linear accelerator will consist of a succession of accelerating structures and heavy electromagnets (quadrupoles). The former are used to accelerate the particles to increase their energy; the latter are used to maintain the beam inside the vacuum chamber (alternating gradient) and to reach the required luminosity at the collision point. However, any oscillation of one quadrupole deflects the beam, and reduces the luminosity. More precisely, if $\Phi_{x}(f)$ is the power spectral density of the vertical displacement of the quadrupole, it has been estimated that the integrated Root Mean Square (RMS) $\sigma_{x}(f)$, defined as

$\sigma_{x}(f)=\sqrt{\int_{f}^{\infty} \Phi_{x}(v) d v}$

must stay below $1 \mathrm{~nm}$ [2] above $1 \mathrm{~Hz}$ to ensure sufficient performances (Fig. 1(a)). Similarly, it must stay below $5 \mathrm{~nm}$ in the lateral direction. This concerns about 2000 quadrupoles per beam line. Additionally, about 80 of these quadrupoles should have the capability to move by steps of some tens of nanometers every $20 \mathrm{~ms}$ [3], with a precision of $\pm 1 \mathrm{~nm}$ (see Fig. 1(b)). To

\footnotetext{
* Corresponding author.

E-mail address: christophe.collette@cern.ch (C. Collette).
}

demonstrate the feasibility to fulfil such stringent requirements, it is planned to build a mock-up of the longest CLIC main beam quadrupole on its support. The paper is organized as follows. Section 2 compares the main strategies studied for the stabilization of quadrupoles in the past. Section 3 presents the concept adopted in this work to support and control a long quadrupole. Section 4 studies in details the stabilization strategy on a simplified quadrupole. Section 5 presents an experimental validation of the stabilization strategy on a scaled test bench. Section 6 draws the conclusions.

\section{Control strategy}

The stabilization of structures at the nanometer scale is a concern in various fields of precision engineering, like interferometers [4], microscopes [5] or manufacturing [6]. In this section, only a few key experiments of quadrupole stabilization are presented, and some of their characteristics are compared in Table 1: the number of degrees of freedom (d.o.f.), the type of actuator, the number of stages for the isolation (i.e. the number of spring-mass systems in series), the positioning capability, the overall stiffness of the support and the ratio between the RMS integrated displacements of the ground $\sigma_{w}$ and the mass to stabilize $\sigma_{x}$ at $1 \mathrm{~Hz}$.

In order to understand the advantages and disadvantages of these approaches, let us consider a single d.o.f. quadrupole shown in Fig. 2(a). Assuming that the alignment stage (which is not discussed in this paper) is completely rigid, the transmissibility $T_{w x}(f)$ between the ground $w$ and the mass 

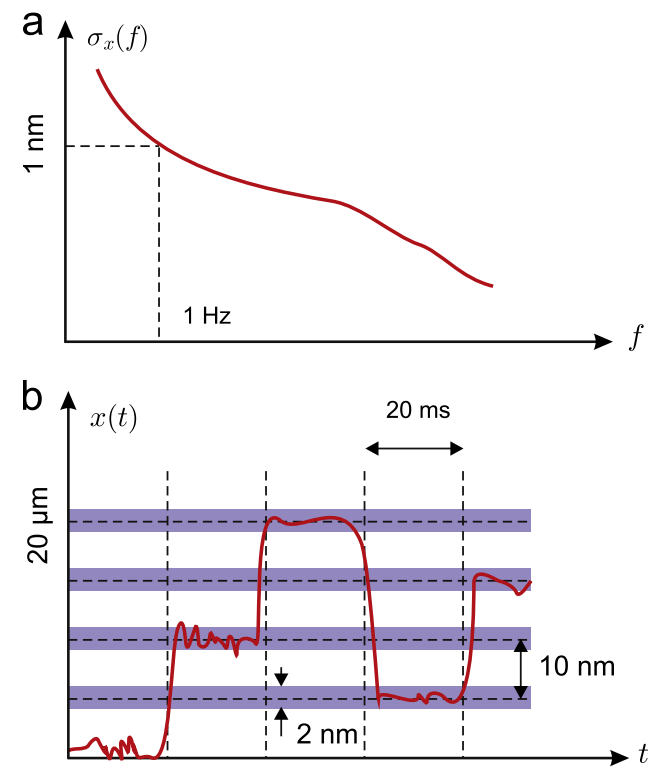

Fig. 1. (a) Frequency domain requirements for the stabilization; (b) time domain requirements for the positioning.

Table 1

Comparison of several strategies adopted for the stabilization of quadrupoles.

\begin{tabular}{lllll}
\hline Institution & DESY & CERN & LAPP & SLAC \\
\hline Ref. & {$[7,8]$} & {$[9]$} & {$[10]$} & {$[11,12]$} \\
d.o.f. & 1 & 6 & 6 & 6 \\
Actuator & Piezo- & Piezo- & Piezo- & Electro- \\
& electric & electric & electric & static \\
Stages & 1 & 2 & 2 & 1 \\
Positioning & No & No & No & No \\
Suspension & Stiff & Soft & Soft & Soft \\
$\sigma_{w} / \sigma_{x}$ at $1 \mathrm{~Hz}$ & 3 & 3 & 2 & 1.5 \\
\hline
\end{tabular}

(quadrupole) displacement $x$ is shown in Fig. 2(b) for three values of the damping coefficient $c$. An overshoot appears on the transmissibility at the resonance frequency of the system. The isolation properties of the support starts at $\sqrt{2} f_{n}$ (where $2 \pi f_{n}=\sqrt{k / m}$ ). Above that frequency, the amplitude of the response is smaller than the amplitude of the excitation. Increasing the damper constant $c$ leads to a reduction of the overshoot, but at the expense of a degradation of the isolation performances. On the other hand, a reduction of the stiffness increases the isolation at low frequencies (Fig. 3(a)). Then, in order to increase the passive isolation, the first idea is to reduce the value of $f_{n}$ as much as possible $[11,12]$. However, as the resonance frequency of the system decreases, it also becomes more sensitive to any external force directly applied on the quadrupole at very low frequency. This is illustrated in Fig. 3(b), showing the transmissibility between a force $F_{a}$ applied on the quadrupole and its vertical displacement $x$. Two stages configurations $[9,10]$ are also based on soft supports. While efficient for the stabilization of quadrupoles, these systems are much too soft to fulfil the positioning requirements (Fig. 1(b)). For this reason, it has been decided to use stiff piezoelectric supports like in Refs. [7,8], and reduce actively the transmissibility $T_{w x}(f)$ at low frequencies (mainly the range between 1 and $20 \mathrm{~Hz}$ ) [13]. a
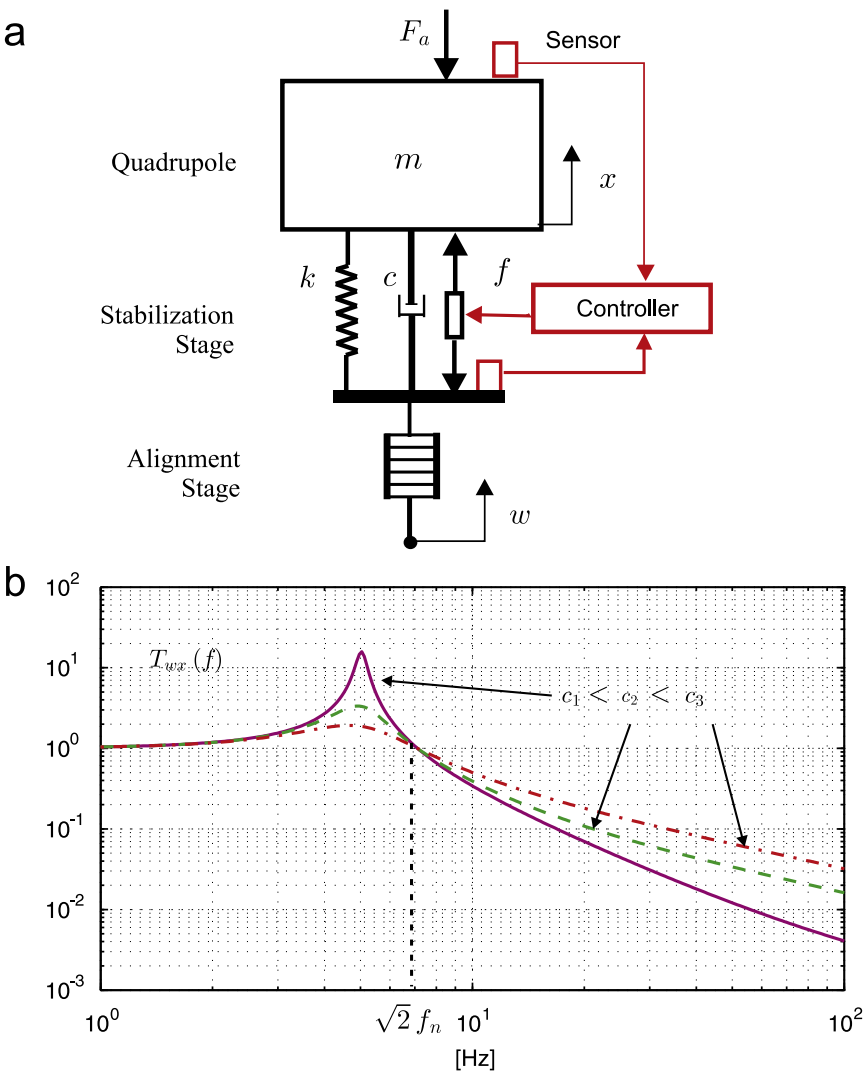

Fig. 2. (a) Single d.o.f oscillator; (b) transmissibility $T_{w x}(f)$ between the ground and the mass displacement for three values of the damping coefficient.

\section{Six d.o.f. quadrupole}

\subsection{Support strategy and system dynamics}

The strategy adopted to provide the required positioning capabilities to the quadrupole is inspired from the concept of a Stewart platform [14-16]. It is a well known concept that has been applied for both vibration isolation and precise positioning of ground and space structures. To tackle with the large length of the quadrupole, the six legs are mounted as depicted in Fig. 4. For reasons of simplicity, it has been decided to fix the orientations of the legs using only two parameters: $\alpha$ fix the orientation in the horizontal plane and $\beta$ the inclination with respect to a vertical axis. Their numerical values result from a tradeoff between the following requirements: provide a good stability in the longitudinal direction, manoeuvrability in both vertical and lateral directions, allow a sufficient resolution in the vertical direction, and ensure a static equilibrium when no control is applied. Assuming that the quadrupole is rigid, the dynamic equations of the system are

$M \ddot{\mathbf{x}}=\mathbf{F}$

where $M=\operatorname{diag}\left(m, m, m, I_{\theta}, I_{\phi}, I_{\psi}\right)$ is the mass matrix, $\mathbf{x}=(x, y, z, \theta$, $\phi, \psi)$ is the vector describing small displacements of the quadrupole, and $\mathbf{F}$ is the vector of resulting forces and torques applied by the legs on the quadrupole. $\mathbf{F}$ is related to the axial forces in each leg by

$\mathbf{F}=B \mathbf{f}$

where $\mathbf{f}=\left(f_{1}, f_{2}, \ldots, f_{6}\right)^{T}$ is the vector of active control forces in the six legs and $B$ the force jacobian matrix. Assuming that there is no 

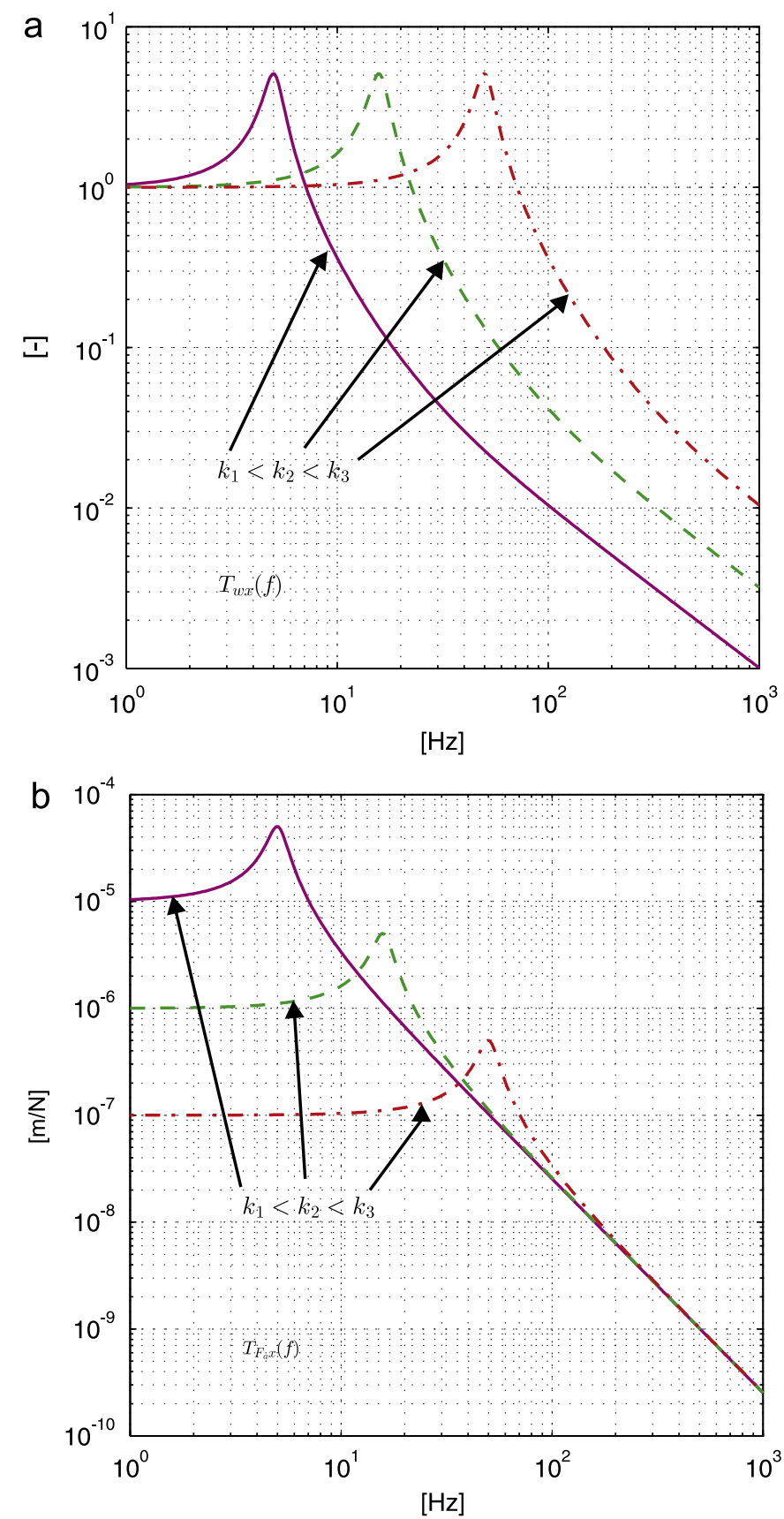

Fig. 3. Effect of the support stiffness on (a) The transmissibility $T_{w x}(f)$ between the ground and the mass displacement; (b) the transmissibility $T_{F_{a} x}(f)$ between a force $F_{a}$ applied on the mass and the mass displacement.

damping in the legs, $f_{i}$ is given by

$f_{i}=k_{a}\left(-q_{i}+\Delta_{i}+w_{i}^{l}\right)$

where $k_{a}$ is the axial stiffness of each leg, $q_{i}$ and $w_{i}^{l}$ are, respectively, the displacement of the quadrupole and the ground in the direction of the leg. $\Delta_{i}$ is the elongation of the leg due to a voltage $V_{i}$ applied to the piezoelectric stack

$\Delta_{i}=n d_{33} V_{i}$

where $n d_{33}$ is a characteristic of the actuator.

Replacing Eqs. (3) and (4) in Eq. (2) gives

$M \ddot{\mathbf{x}}+K \mathbf{x}=k_{a} B \Delta+k_{a} B \mathbf{w}^{l}$
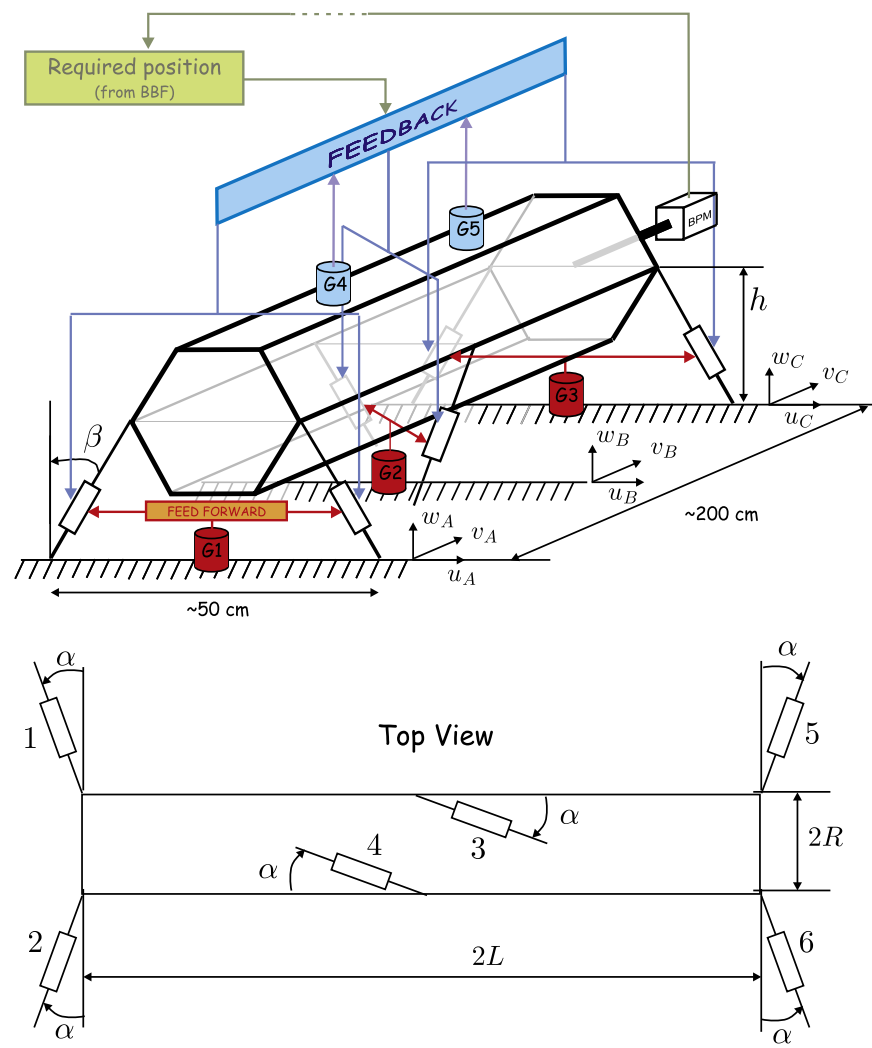

Fig. 4. Simplified drawing of the quadrupole.

or again

$M \ddot{\mathbf{x}}+K \mathbf{x}=k_{a} B \Delta+k_{a} B E \mathbf{w}$

where $K=k_{a} B B^{T}$ is the stiffness matrix, $\mathbf{w}$ and $\mathbf{w}^{l}$ are the ground excitation vector and the ground excitation vector in the legs, and $E$ is the excitation matrix projecting $\mathbf{w}$ in the directions of the legs.

Let $J$ be the Jacobian matrix relating the elongations velocities of the legs $\dot{\mathbf{q}}$ and the velocity vector $\dot{\mathbf{x}}$ as $\dot{\mathbf{q}}=J \dot{\mathbf{x}}$. According to the virtual work principle, we have

$\mathbf{F}^{T} \delta \mathbf{x}=\mathbf{f}^{T} \delta \mathbf{q}=\mathbf{f}^{T} J \delta \mathbf{x}$

After identification, we have $\mathbf{F}=J^{T} \mathbf{f}$. Comparing with Eq. (3) leads to $B=J^{T}$. The analytical expression of $J$ is found as follows. First, let us split the velocity vector $\dot{\mathbf{x}}$ into translational and rotational components such as $\dot{\mathbf{x}}^{T}=\left(\mathbf{v}^{T}, \omega^{T}\right)$ where $\mathbf{v}^{T}=(\dot{x}, \dot{y}, \dot{z})$ and $\boldsymbol{\omega}^{T}=(\dot{\theta}, \dot{\phi}, \dot{\psi})$. Then, the velocity of the fixation point of leg $i$ is

$\mathbf{v}_{i}=\mathbf{v}+\omega \times \mathbf{p}_{i}$

where $\mathbf{p}_{i}$ is the coordinate of the extremity of leg $i$ in the reference frame fixed on the quadrupole. If $\mathbf{1}_{i}$ is a unit vector in the direction of leg $i$, the velocity of the extension of the leg is obtained by projecting $\mathbf{v}_{i}$ along $\mathbf{1}_{i}$

$\dot{q}_{i}=\mathbf{1}_{i}^{T} \mathbf{v}_{i}=\mathbf{1}_{i}^{T}\left(\mathbf{v}+\omega \times \mathbf{p}_{i}\right)$

or

$\dot{q}_{i}=\mathbf{1}_{i}^{T} \mathbf{v}_{i}=\mathbf{1}_{i}^{T}\left(\mathbf{v}-\mathbf{p}_{i} \times \omega\right)$.

Proceeding the same way for each leg, we have finally

$J=\left(\begin{array}{cc}\ldots & \ldots \\ \mathbf{1}_{i}^{T} & -\mathbf{1}_{i}^{T} \tilde{\mathbf{p}}_{i} \\ \ldots & \ldots\end{array}\right)$

where $\tilde{\mathbf{p}}_{i}$ is the antisymmetric matrix calculated from $\mathbf{p}_{i}$ to express the cross product. In details, the matrices of unit vector $Q=\left(\mathbf{1}_{1}, \ldots, \mathbf{1}_{6}\right)$ and positions of legs extremity $P=\left(\mathbf{p}_{1}^{T}, \ldots, \mathbf{p}_{6}^{T}\right)$ are, 
respectively,

$Q^{T}=\left(\begin{array}{ccc}\sin \beta \cos \alpha & \sin \beta \sin \alpha & \cos \beta \\ -\sin \beta \cos \alpha & \sin \beta \sin \alpha & \cos \beta \\ -\sin \beta \sin \alpha & -\sin \beta \cos \alpha & \cos \beta \\ \sin \beta \sin \alpha & \sin \beta \cos \alpha & \cos \beta \\ \sin \beta \cos \alpha & -\sin \beta \sin \alpha & \cos \beta \\ -\sin \beta \cos \alpha & -\sin \beta \sin \alpha & \cos \beta\end{array}\right)$

and

$P=\left(\begin{array}{cccccc}-R & R & -R & R & -R & R \\ -L & -L & 0 & 0 & L & L \\ h & h & h & h & h & h\end{array}\right)$.

\subsection{Seismic response calculation}

Because the distance between any two legs (not only located side by side) does not exceed a few meters, their excitation in any particular direction should be coherent. Thus, it is assumed that the excitation is the same for each pair of legs having the same coordinate along the main quadrupole axis. That is, the excitation vector is $\mathbf{w}=\left(u_{A}, v_{A}, w_{A}, u_{B}, v_{B}, w_{B}, u_{C}, v_{C}, w_{C}\right)^{T}$. Also, as the coherence between the ground motion in perpendicular directions is very low, the excitation matrix can be approximated by the following bloc diagonal form

$E^{T}=\left(\begin{array}{cccccc}\mathbf{1}_{1} \cdot \mathbf{e}_{x} & \mathbf{1}_{2} \cdot \mathbf{e}_{x} & 0 & 0 & 0 & 0 \\ \mathbf{1}_{1} \cdot \mathbf{e}_{y} & \mathbf{1}_{2} \cdot \mathbf{e}_{y} & 0 & 0 & 0 & 0 \\ \mathbf{1}_{1} \cdot \mathbf{e}_{z} & \mathbf{1}_{2} \cdot \mathbf{e}_{z} & 0 & 0 & 0 & 0 \\ 0 & 0 & \mathbf{1}_{3} \cdot \mathbf{e}_{x} & \mathbf{1}_{4} \cdot \mathbf{e}_{x} & 0 & 0 \\ 0 & 0 & \mathbf{1}_{3} \cdot \mathbf{e}_{y} & \mathbf{1}_{4} \cdot \mathbf{e}_{y} & 0 & 0 \\ 0 & 0 & \mathbf{1}_{3} \cdot \mathbf{e}_{z} & \mathbf{1}_{4} \cdot \mathbf{e}_{z} & 0 & 0 \\ 0 & 0 & 0 & 0 & \mathbf{1}_{5} \cdot \mathbf{e}_{x} & \mathbf{1}_{6} \cdot \mathbf{e}_{x} \\ 0 & 0 & 0 & 0 & \mathbf{1}_{5} \cdot \mathbf{e}_{y} & \mathbf{1}_{6} \cdot \mathbf{e}_{y} \\ 0 & 0 & 0 & 0 & \mathbf{1}_{5} \cdot \mathbf{e}_{z} & \mathbf{1}_{6} \cdot \mathbf{e}_{z}\end{array}\right)$

Ground vibration measurements have been compared in Ref. [17], and led to the choice of a quiet place to perform the stabilization experiments. Fig. 5(a) shows the power spectral densities of the ground displacement at that place, and Fig. 5(b) shows the coherence between $w_{A}(t)$ and $w_{C}(t)$ (located $2 \mathrm{~m}$ apart). Without active control, the stationary response of the quadrupole is given in the Fourier domain by

$X(f)=T(f) W(f)$

where $T(f)=\left[-M(2 \pi f)^{2}+K\right]^{-1} E$ and the random response is

$\Phi_{x}(f)=T(f) \mathbf{S}_{w}(f) T^{*}(f)$

where $\mathbf{S}_{w}(f)$ is the ground excitation matrix [18] given by

$\mathbf{S}_{w}(f)=\left(\begin{array}{ccccccccc}\Phi_{u}(f) & 0 & 0 & \Phi_{u}(f) & 0 & 0 & \Phi_{u}(f) & 0 & 0 \\ 0 & \Phi_{v}(f) & 0 & 0 & \Phi_{v}(f) & 0 & 0 & \Phi_{v}(f) & 0 \\ 0 & 0 & \Phi_{w}(f) & 0 & 0 & \Phi_{w}(f) & 0 & 0 & \Phi_{w}(f) \\ \Phi_{u}(f) & 0 & 0 & \Phi_{u}(f) & 0 & 0 & \Phi_{u}(f) & 0 & 0 \\ 0 & \Phi_{v}(f) & 0 & 0 & \Phi_{v}(f) & 0 & 0 & \Phi_{v}(f) & 0 \\ 0 & 0 & \Phi_{w}(f) & 0 & 0 & \Phi_{w}(f) & 0 & 0 & \Phi_{w}(f) \\ \Phi_{u}(f) & 0 & 0 & \Phi_{u}(f) & 0 & 0 & \Phi_{u}(f) & 0 & 0 \\ 0 & \Phi_{v}(f) & 0 & 0 & \Phi_{v}(f) & 0 & 0 & \Phi_{v}(f) & 0 \\ 0 & 0 & \Phi_{w}(f) & 0 & 0 & \Phi_{w}(f) & 0 & 0 & \Phi_{w}(f)\end{array}\right)$

where $\Phi_{i_{A}}(f)=\Phi_{i_{B}}(f)=\Phi_{i_{C}}(f)=\Phi_{i}(f)$, and $i=\{u, v, w\}$. For simplicity, the active stabilization is studied in the next section on a three d.o.f. system.

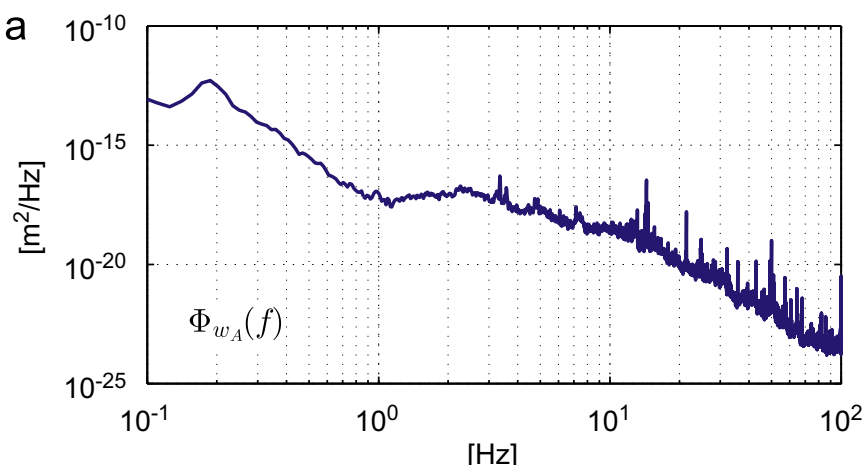

$[\mathrm{Hz}]$

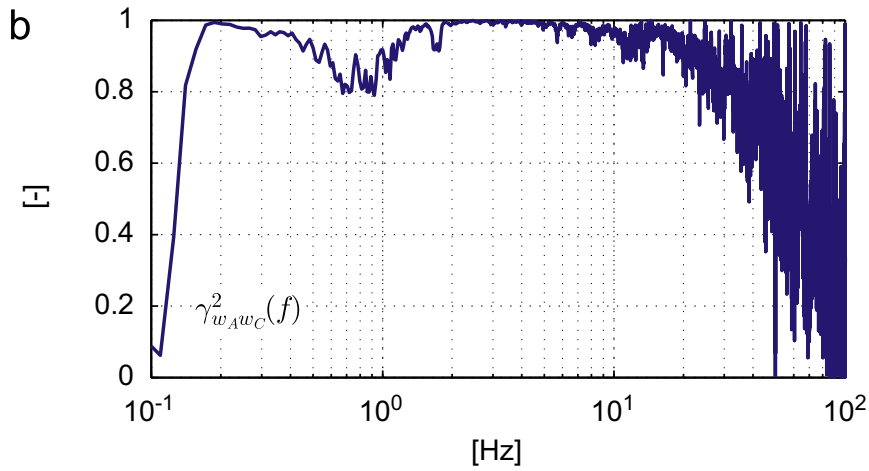

Fig. 5. (a) Power spectral density of the ground displacement $\Phi_{w_{A}}(f)$; (b) coherence of the vertical displacement between two points $w_{A}(t)$ and $w_{c}(t)$.

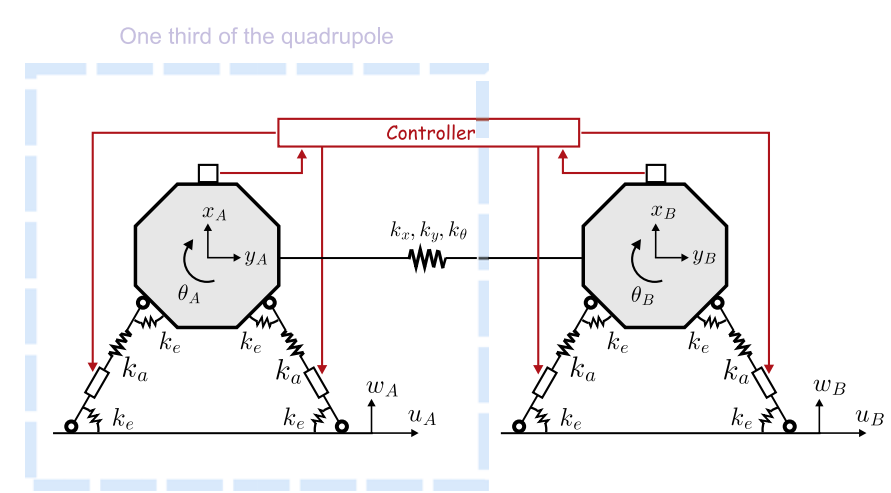

Fig. 6. Control strategy used for the stabilization.

\section{Quadrupole stabilization}

Let us start with one third of the quadrupole, mounted on two active legs, like represented in the left part of Fig. 6 .

After integrating the lateral and vertical ground velocities measured by the geophone, there is a linear relationship between the sensor output and the actuator input

$\mathbf{y}=C \mathbf{x}$

where $\mathbf{y}$ is the measurement vector, $C$ a matrix containing the sensor dynamics, and $\mathbf{x}=\left(x_{A}, y_{A}, \theta_{A}\right)$. In this configuration, the geophone cannot provide any information on $\theta_{A}$, i.e. there is a null column in matrix $C$. Focusing on the observable subspace (with a 0 index)

$\mathbf{y}_{0}=C_{0} \mathbf{x}_{0}$

or

$\mathbf{y}_{0}=C_{0} J_{0}^{-1} \mathbf{q}_{0}$. 


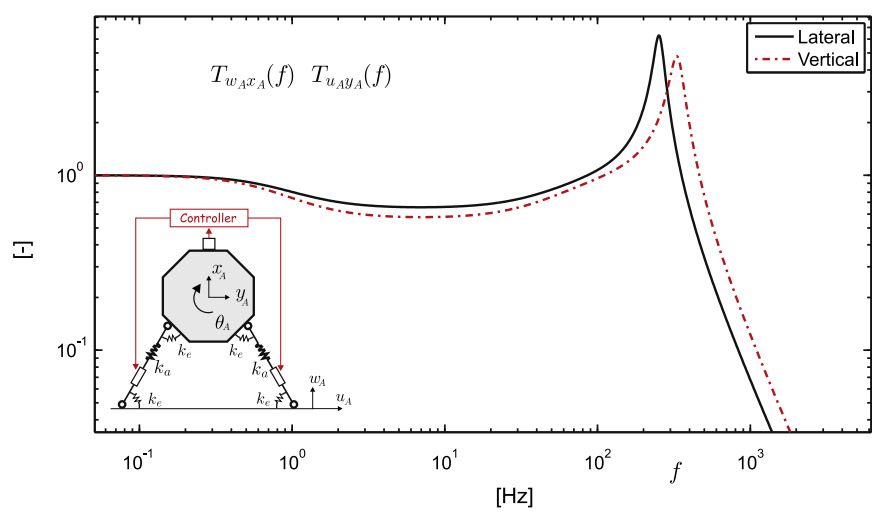

Fig. 7. Active stabilization in both vertical and lateral directions using a SVD controller.

Additionally, $C_{0} J_{0}^{-1}$ can be decomposed into singular values

$\mathbf{y}_{0}=U \Sigma V^{T} \mathbf{q}_{0}$

where $\Sigma=\operatorname{diag}\left(\sigma_{1}, \sigma_{2}\right)$. Then, assuming that the flexible resonances are above the frequency range of interest, the controller is simply found using Eq. (6) and inverting Eq. (18)

$\mathbf{F}_{b}=k_{a} B \Delta=k_{a} B V H(f) \Sigma^{-1} U^{T} \mathbf{y}$

where $H(f)=\operatorname{diag}\left(h_{1}(f), h_{2}(f)\right)$ is the gain vector. Here, the singular values are used as a tool for the design of the system. For example, vertical legs $(\beta=0)$ will result in a null singular value for the lateral direction, i.e. a configuration that cannot be controlled. The gains can be tuned independently, or chosen all the same. The latter case is illustrated in Fig. 7 showing the transmissibilities $T_{w_{A} x_{A}}(f)$ and $T_{u_{A} y_{A}}(f)$ between the ground and the quadrupole, respectively, in the vertical and lateral direction. In this case, the quadrupole is stabilized in both directions using a scalar controller. In the simulation, the following numerical values have been used for the legs: $k_{a}=3 \mathrm{e} 8 \mathrm{~N} / \mathrm{m}, k_{e}=588 \mathrm{Nm} / \mathrm{rad}, \beta=\pi / 6$, and a mass of $420 / 3 \mathrm{~kg}$ and inertia of $15.6 \mathrm{~kg} \mathrm{~m}^{2}$ for the quadrupole.

Now the question is: can such a local (decentralized) controller be applied to each pairs of legs to stabilize the entire quadrupole? In order to answer this question, let us consider the system depicted in Fig. 8(a), which is a simplified version of Fig. 6, with only one leg below each mass. The dynamics of the system is still governed by Eq. (7), where $\mathbf{x}=\left(x_{A}, x_{B}\right)^{T} ; \Delta=\left(\delta_{A}, \delta_{B}\right)^{T} ; \mathbf{w}=\left(w_{A}, w_{B}\right)^{T}$;

$M=\left(\begin{array}{cc}m_{A} & 0 \\ 0 & m_{B}\end{array}\right) ; \quad K=\left(\begin{array}{cc}k_{a}+k & -k \\ -k & k_{a}+k\end{array}\right) ; \quad B=E=\left(\begin{array}{cc}1 & 0 \\ 0 & 1\end{array}\right)$.

In this case, one controller is used for each leg: $\delta_{A}=g_{A} x_{A}$; $\delta_{B}=g_{B} x_{B}$. The global stability of the quadrupole is represented by the relative displacement $d$ between the two masses, defined by $d=x_{A}-x_{B}$. This variable is essentially affected by the coherence between $w_{A}$ and $w_{B}$ : the lower is the coherence, the higher is the excitation of $d$. Thus, let us consider that the system is subjected to the following excitation matrix

$\mathbf{S}_{w}(f)=\left(\begin{array}{ll}1 & \varepsilon \\ \varepsilon & 1\end{array}\right)$

where $\varepsilon$ is a parameter comprised between 0 and 1 . If $\varepsilon=1$ (like in Fig. 5(b)), $w_{A}$ and $w_{B}$ are fully coherent; If $\varepsilon=0, w_{A}$ and $w_{B}$ are fully incoherent. Figs. 8(b) and (c) show the power spectral density of $x_{A}$ and $d$ for, respectively, $\varepsilon=0.9$ and $\varepsilon=0\left(\Phi_{x_{B}}(f)\right.$ is the same as $\Phi_{x_{A}}(f)$ because of the symmetry of the system). The following numerical values have been used for the simulation: $m_{A}=m_{B}=420 / 6 \mathrm{~kg}, k_{a}=200 \mathrm{MN} / \mathrm{m}, k=172 \mathrm{MN} / \mathrm{m}$. As expected, one sees on Fig. 8 that, the lower the value of $\varepsilon$, the level of $\Phi_{d}(f)$. a
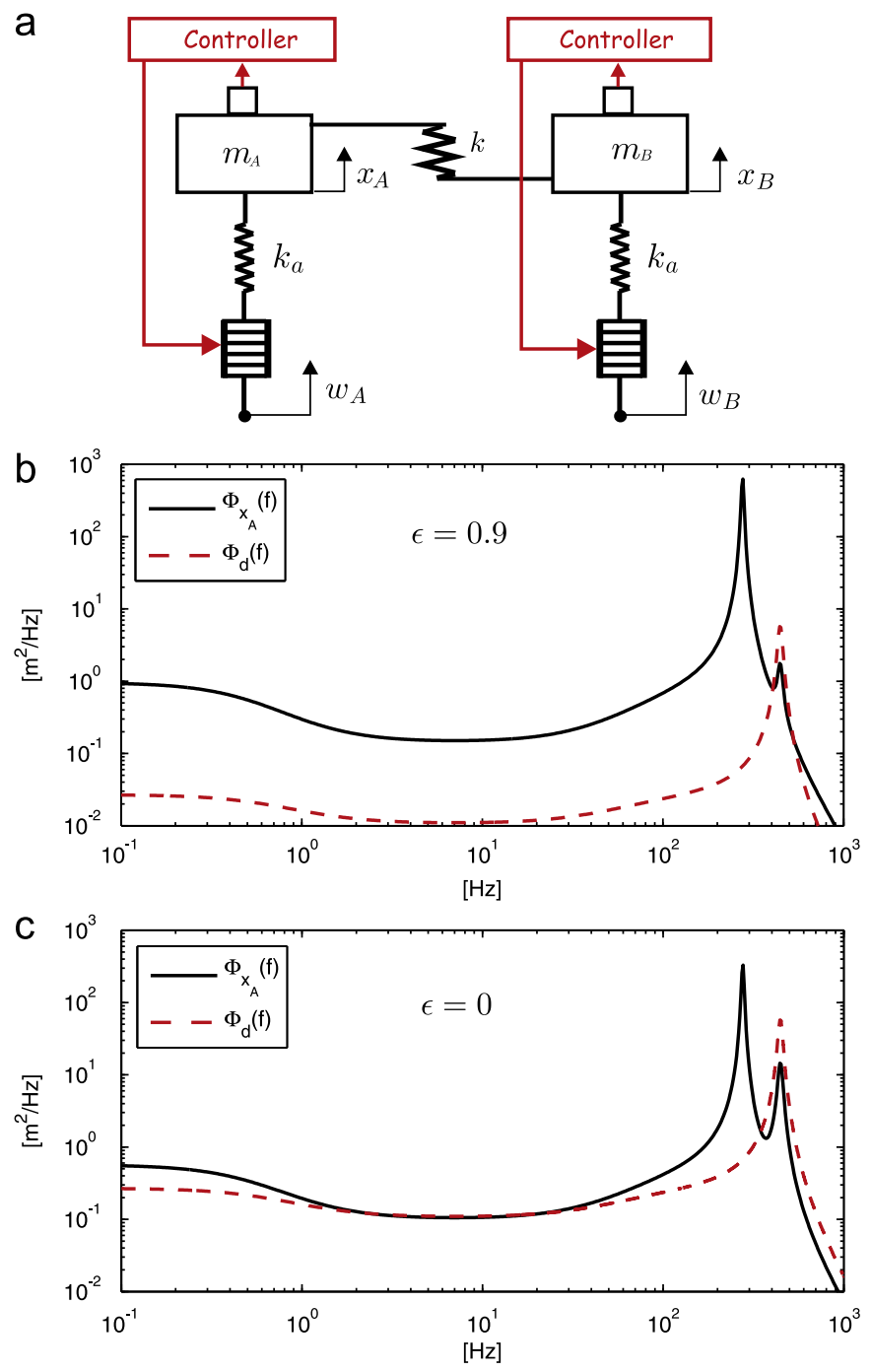

Fig. 8. Two-masses quadrupole on two active legs: (a) scheme of the model; (b) $\Phi_{X_{A}}(f)$ and $\Phi_{d}(f)$ for a coherent excitation $(\varepsilon=0.9)$; (c) $\Phi_{X_{A}}(f)$ and $\Phi_{d}(f)$ for a incoherent excitation $(\varepsilon=0)$.

However, one also sees that, whatever the value of $\varepsilon$, the action of the controller always to decrease both the amplitude of $\Phi_{x_{A}}(f)$ and $\Phi_{d}(f)$. In other words, the simple decentralized controller presented in this section stabilize both parts of the quadrupole, and at the same time, improves its global stability.

\section{Test bench}

\subsection{Modeling}

In order to test experimentally the stabilization strategy described above, a scaled test bench has been designed to represent one sixth of the quadrupole mounted on one active leg (Fig. 9(a)). The experimental setup consists of a guided piezoelectric stack, clamped in a double membrane like structure to allow only a vertical motion (Fig. 9(b)). Two geophones are used to measure the vibrations at both ends of the actuator. The aim of the experiment is to stabilize a small mass laying on the top of the membrane, i.e. the geophone itself.

The dynamic equation of the full scale model depicted in Fig. 9(a) is

$m \ddot{x}+k(x-w-\Delta \cos \beta)=0$ 

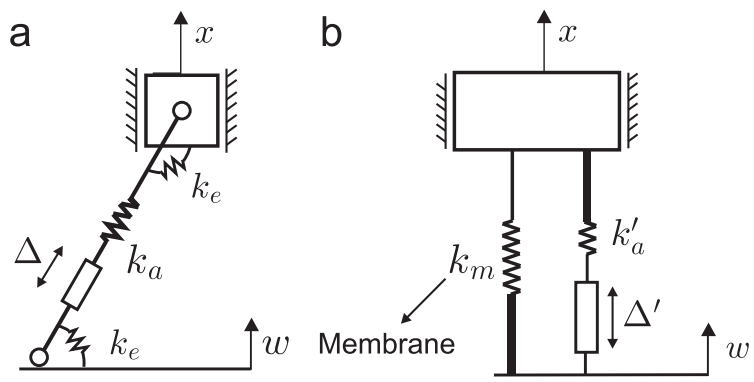

Fig. 9. (a) One sixth of the quadrupole on one active leg; (b) scaled setup.

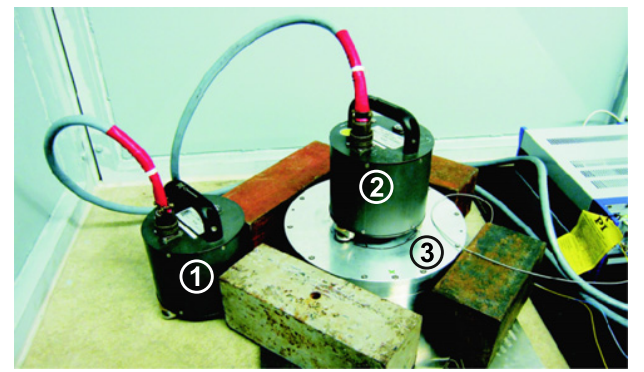

1. Geophone $\dot{w}$

2. Geophone $\dot{x}$

3. Guide

Fig. 10. Picture of the experimental setup.

where $m=420 / 6=70 \mathrm{~kg}$, and the stiffness of the actuator $k_{a}$ and the jointures $k_{e}$ are lumped in $k$, which is found to be $k=250 \mathrm{MN} /$ $\mathrm{m} ; x$ the vertical displacement of the quadrupole on the top of the actuator, $w$ the ground motion, and

$\Delta=n d_{33} V$

the elongation of the piezoelectric actuator induced by a voltage $V$ [19].

The scaled setup will be representative of the full scale system if its dynamics is governed by the same equation, i.e. if

$\frac{k}{m}=\frac{k^{\prime}}{m^{\prime}}$

where $m^{\prime}=2.5 \mathrm{~kg}$ is the mass of a geophone (the mass of the membrane is much smaller than the mass of the geophone), and $k^{\prime}$ is the sum of the membrane stiffness $k_{m}$ and the small actuator stiffness $k_{a}^{\prime}$.

Assuming that the vertical stiffness of the guide is negligible with respect to the stiffness of the actuator, the same dynamics is obtained by taking an actuator with a stiffness $k^{\prime}=25 \mathrm{MN} / \mathrm{m}$. The experimental setup is shown in Fig. 10. It is located in a tunnel where the amplitude of the ground motion is similar to the values measured in the LHC tunnel.

For frequencies below the resonance of the geophone on the stiffness of the actuator, two ways to decrease the transfer function $T_{w x}(f)$ between the two geophones can be considered:

(i) Integrate the signal of the top geophone in a feedback loop:

$$
F_{b}=k \Delta=K_{p}^{b} x
$$

(ii) Integrate the signal of the ground geophone in a feed forward strategy:

$$
F_{f}=k \Delta=K_{p}^{f} w
$$

where $K_{p}^{b}$ and $K_{p}^{f}$ are the gain of the controllers. Theoretically, both of these strategies are efficient. However, in this study, the first one is preferred because the performances of the feed forward are limited by the coherence between the ground vibration

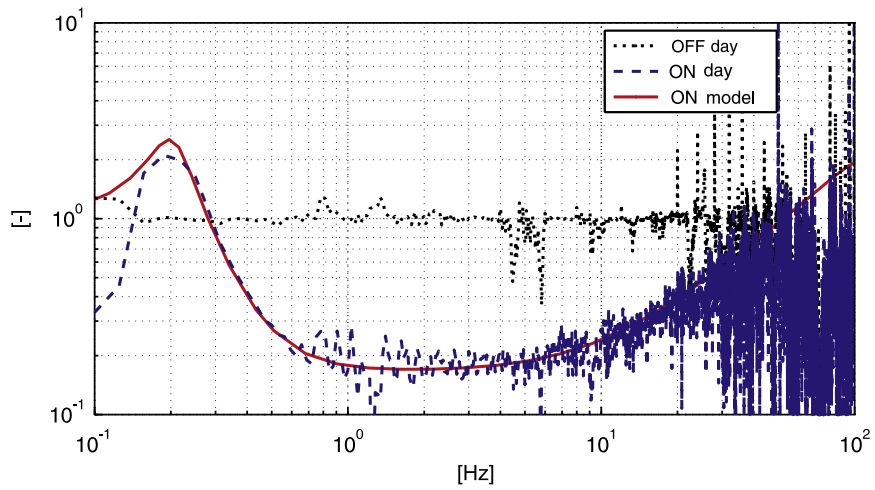

Fig. 11. Transfer function $T_{w x}(f)$ calculated (solid line), measured during the day when the controller is OFF (dotted line) and ON (dashed line).

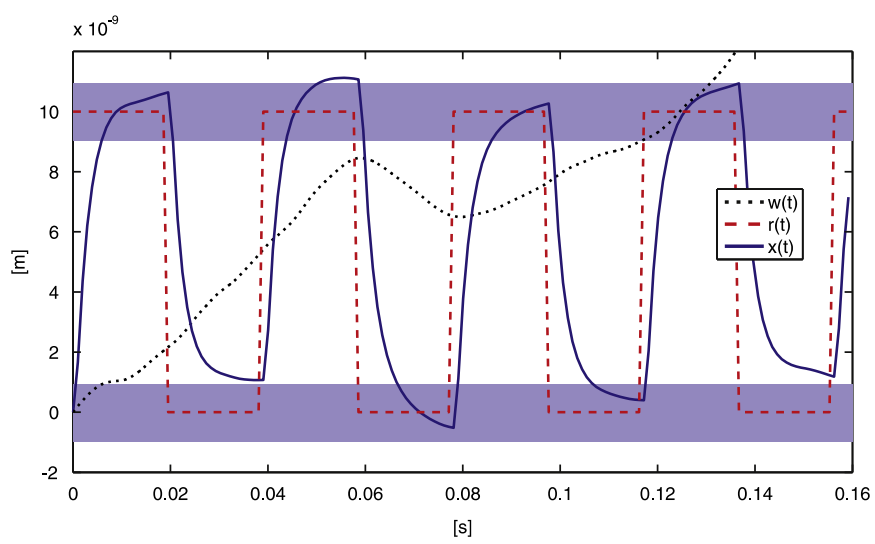

Fig. 12. Simulation of the time history of the geophone motion $x(t)$, subjected to a ground excitation $w(t)$ and a requested position $r(t)$.

measurement and the uncontrolled output $x$. Fig. 11 shows the transmissibility $T_{w x}(f)$ using the position feedback strategy. A Butterworth high pass filter at $0.5 \mathrm{~Hz}$ is introduced in the controller to remove the drift in the signals, and a lag at $30 \mathrm{~Hz}$ to improve the stability. In order to test if the top geophone can be also positioned to a requested position $r$, the feedback control law has been transformed into a classical Proportional Integral Derivative (PID) controller. Eqs. (24) and (25) become

$F_{b}=K_{d}^{b} \dot{x}+K_{p}^{b}(r-x)+K_{i}^{b} \int(r-x) d t$

$F_{f}=K_{d}^{f} \dot{w}+K_{p}^{f} w$

where $K_{d}^{b}, K_{i}^{b}, K_{d}^{f}$ are the gains of the controller, and $r(t)$ is the requested position.

Fig. 12 shows a short time history simulated with the control law, when the requested position is a square function with an amplitude of $10 \mathrm{~nm}$ and steps of $20 \mathrm{~ms}$.

The following section presents the experimental results obtained for the stabilization.

\subsection{Experiments}

The piezo-actuator is a $\mathrm{P}-753.21 \mathrm{C}$ [20]. It works in a closed loop configuration together with a built-in capacitive sensor and an amplifier (Fig. 13). The calibration factor for the piezo actuator system is $400 \mu \mathrm{V} / \mathrm{nm}$. In the closed loop configuration the actuator has a maximum stroke of $25 \mu \mathrm{m}$ and a resolution of $0.1 \mathrm{~nm}$. The geophones (Guralp CMG-6T [21]) have a differential 


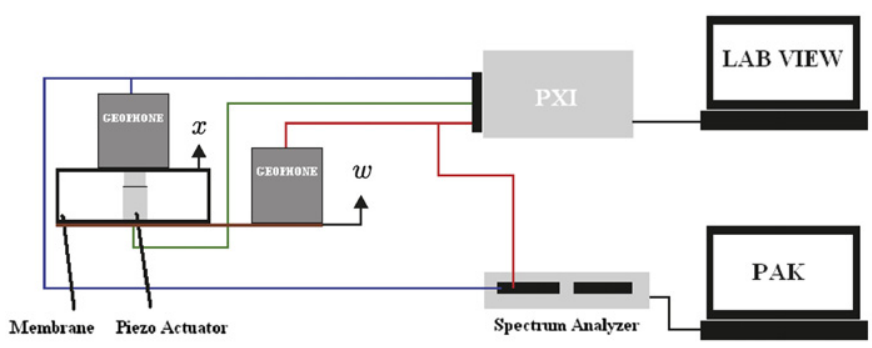

Fig. 13. Description of the experimental setup.

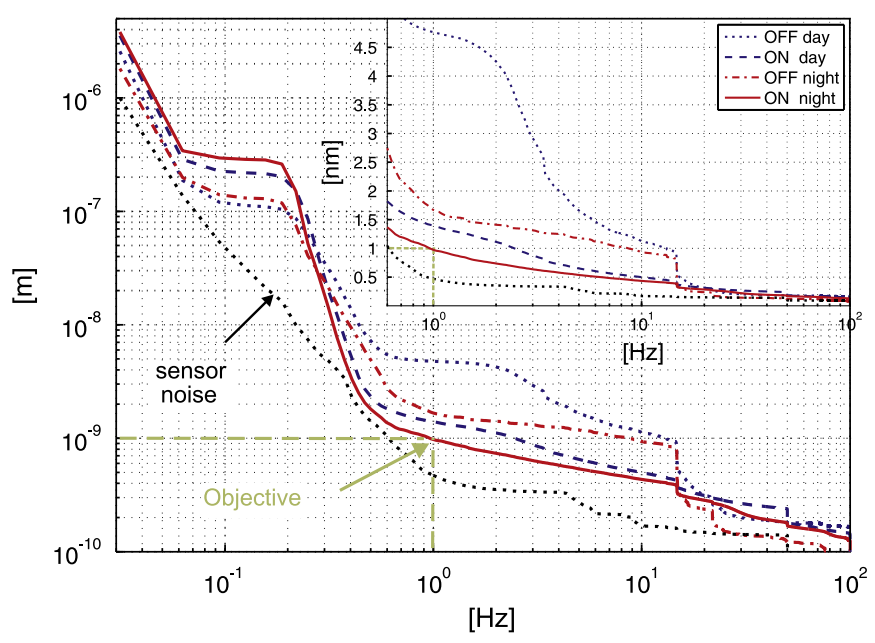

Fig. 14. Comparison of RMS integrated of the top displacement $\sigma_{x}(f)$ when the controller is ON and OFF, during the day and during the night.

sensitivity of about $2000 \mathrm{~V} /(\mathrm{m} / \mathrm{s})$ and a frequency range between $30 \mathrm{~s}$ and $100 \mathrm{~Hz}$. The real time control system is based on a card PXI-6289 [22] for data acquisition. The characteristics of the card are as follows: 32 single ended or 16 differential input channels (18 bit resolution), four analog outputs (16 bit resolution).

The same filters are used as in the simulation. The range of the signal for the piezo-electric actuator has the low limit of $-2 \mathrm{~V}$. In order to protect the actuator and to ensure that the signal is not exceeding this limit, an offset of $1 \mathrm{~V}$ is added to the output signal of the control system. The sampling rate of the card is guaranteed by the producer and can be set up to $625 \mathrm{kSample} / \mathrm{s}$. On the other hand, the program loop rate can vary depending on many factors (CPU load, amount of calculations in the loop, amount of information to be updated during the loop run). These two processes have to be well synchronized. In order to evaluate the performances of the stabilization, the signals from the geophones are sent to a second acquisition system in parallel with the PXI. This system has a better resolution, and better performances are delivered by the PXI when it does not save data. The system used was the MKII made by Müller-BBM. It contains 16 input channels with DSP (Digital Spectrum Processing) for each four channels with a sampling frequency up to $204 \mathrm{kHz}$. The dynamic resolution is 24 bits, and it can be used in a range between $10 \mathrm{mV}$ and $50 \mathrm{~V}$ with a noise level lower than $1 \mathrm{pV}$. The transfer function $T_{w x}(f)$ measured during the day is compared with the theoretical curve in Fig. 11. It has been calculated by

$T_{w x}(f)=\frac{\Phi_{x w}(f)}{\Phi_{w w}(f)}$.

The transfer function measured when the controller is OFF is also shown in the same Figure to demonstrate the effect of the feedback operation. Fig. 14 shows the corresponding RMS integrated displacement calculated from $\Phi_{x}(f)$. At $1 \mathrm{~Hz}$, one sees that the feedback control has reduced $\sigma_{x}(f)$ from 4.7 to $1.4 \mathrm{~nm}$, i.e. a reduction by a factor 3.5 . The same experiment has been also conducted during the night, when the ground motion is even lower. In this case, $\sigma_{x}$ is reduced from 1.8 to $1 \mathrm{~nm}$ at $1 \mathrm{~Hz}$. Although this value corresponds to the requirements (Fig. 1(a)), the controller still needs to be improved to reach this required value during the day, i.e. in conditions similar to a realistic, active accelerator environment. Better results are anyway expected from an optimized combination of the feedback and feed forward, and a more adapted hardware.

\section{Conclusions and future work}

First of all, some key experiments of quadrupole stabilization have been compared. In order to fulfil both the stabilization and positioning requirements, it has been chosen to mount the structure on stiff supports. Then, a concept of six legs derived from a Stewart platform has been presented. The stabilization strategy has been studied numerically on a simplified model of the quadrupole. It has been shown that a SVD controller can be applied to stabilize the quadrupole in both the vertical and the lateral direction. Additionally, it has been shown that a decentralized strategy improves also the global stability of the structure.

Finally, the stabilization strategy has been validated experimentally on a scaled single d.o.f. set-up. This experiment has shown that the RMS integrated displacement is reduced by a factor 3.5 at $1 \mathrm{~Hz}$ during the day, and by nearly a factor 2 during the night, leading to the required value of $\sigma_{x}=1 \mathrm{~nm}$ at $1 \mathrm{~Hz}$. This also shows that, although better results are expected from a more powerful hardware, the current equipment is already capable to work at this high level of precision.

Before stabilizing and positioning experimentally the long quadrupole, the next step will be to consider first a heavy compact object, in order to address all the difficulties of the stabilization and positioning of the long quadrupole (except its flexibility), which are mainly load compensation, jointure design, control optimization.

\section{Acknowledgements}

The authors gratefully acknowledge the European Union for funding this research under the seventh framework programme (project EUCARD).

\section{References}

[1] G. Riddone, D. Schulte, H. Mainaud-Durand, I. Syratchev, W. Wuensch, R. Zennaro, R. Nousiainen, A. Samoshkin, Technical specification for the CLIC two-beam module, in: Proceedings of the EPAC 08, Genoa, Italy, 2008.

[2] 〈http://clic-stability.web.cern.ch/clic-stability/〉.

[3] D. Schulte, Beam based alignment in the new clic main linac, in: Proceedings of the 2009 Particle Accelerator Conference, Vancouver, Canada, 2009.

[4] A. Stochino, B. Abbot, Y. Aso, M. Barton, A. Bertolini, V. Boschi, D. Coyne, R. DeSalvo, C. Galli, Y. Huang, A. Ivanov, S. Marka, D. Ottaway, V. Sannibale, C. Vanni, H. Yamamoto, S. Yoshida, Nuclear Instruments and Methods in Physics Research A 598 (2009) 737.

[5] A. Carter, G. King, T. Ulrich, W. Hasley, D. Alchenberger, T. Perkins, Applied Optics 46 (3) (2007) 421.

[6] K. Furutani, M. Suzuki, R. Kudoh, Measurement Science and Technology 15 (2004) 467.

[7] C. Montag, Nuclear Instruments and Methods in Physics Research A 378 (1996) 369.

[8] C. Montag, Active stabilization of mechanical quadrupole vibrations in a linear collider test facility, Ph.D. Thesis, Hamburg University, 1996. 
[9] S. Redaelli, Stabilization of nanometer-size particle beams in the final focus system of the Compact Linear Collider CLIC, Ph.D. Thesis, University of Lausanne, 2004.

[10] B. Bolzon, Etude des vibrations et de la stabilisation à l'échelle sousnanométreique des doublets finaux d'un collisionneur linéaire, Ph.D. Thesis, University of Savoie, 2007.

[11] S. Allison, L. Eriksson, L. Hendrickson, T. Himel, A. Seryi, Active vibration suppression $\mathrm{r}+\mathrm{d}$ for the next linear collider, in: Proceedings of the Particle Accelerator Conference, Chicago, 2001.

[12] J. Frisch, A. Chang, V. Decker, E. Doyle, L. Eriksson, L. Hendrickson, T. Himel, T. Markiewicz, R. Partidge, A. Seryi, Vibration stabilization of a mechanical model of a x-band linear collider final focul magnet, in: 22nd International Linear Collider Conference, Germany, 16-18 August, 2004.

[13] K. Artoos, O. Capatina, C. Collette, M. Guinchard, C. Hauviller, F. Lackner, J. Pfingstner, H. Schmickler, M. Sylte, M. Fontaine, B. Bolzon, L. Brunetti, G. Deleglise, N. Geoffroy, A. Jeremie, Study of the stabilization to the nanometre level of mechanical vibrations of the CLIC main beam quadrupoles, in: Proceedings of the 2009 Particle Accelerator Conference, Vancouver, Canada, 2009.
[14] J. Spanos, Z. Rahman, G. Blackwood, A soft 6-axis active vibration isolator, in: Proceedings of the American Control Conference, Seattle, Washington, 1995.

[15] A. Hanieh, Active isolation and damping of vibrations via Stewart platform, Ph.D. Thesis, University of Brussels, 2004.

[16] A. Preumont, Vibration Control of Active Structures. An Introduction, Kluwer Academic Publishers, Dordrecht, The Netherlands, 2002.

[17] K. Artoos, O. Capatina, C. Collette, M. Guinchard, C. Hauviller, M. Sylte, B. Bolzon, A. Jeremie, Ground vibration and coherence length measurements for the CLIC nano-stabilization studies, in: Proceedings of the 2009 Particle Accelerator Conference, Vancouver, Canada, 2009.

[18] A. Preumont, Random Vibration and Spectral Analysis, Kluwer Academic Publishers, Dordrecht, The Netherlands, 1994.

[19] A. Preumont, Mechatronics, Dynamics of Electromechanical and Piezoelectric Systems, Springer, Dordrecht, The Netherlands, 2006.

[20] P.I., Physik Instruments catalogue.

[21] Guralp, Guralp catalogue.

[22] N.I., National Instruments catalogue. 